\title{
Reconfigurable LNA for MB-OFDM Receiver using Active Inductor
}

\author{
G.Vinodhini \\ PG Student \\ Dept. of ECE
}

SSN College of Engineering, Chennai, India

\author{
J.Raja \\ Professor \\ Dept. of ECE \\ Sairam Engineering College, Chennai, India
}

\author{
V.Vaithianathan \\ Assistant Professor \\ Dept. of ECE \\ SSN College of Engineering, Chennai, India \\ R.Srinivasan
Professor
Dept. of IT \\ SSN College of Engineering, Chennai, India
}

\begin{abstract}
The Low Noise Amplifier (LNA) is very much important in achieving good reception sensitivity in the wideband transceiver of Ultra Wide Band (UWB) architecture. In this paper, the LNA is designed for Multi Band-Orthogonal Frequency Division Multiplexing (MB-OFDM) receiver, which is used to transmit the information on 14 different sub bands of UWB with minimum of $528 \mathrm{MHz}$ frequency in each band. The LNA comprises of three stages namely input stage, core stage and output stage. The input stage is for better Input matching and output stage contributes better Output matching by acting as a buffer. The core stage is responsible for high gain of the LNA. Different sub bands are achieved with the help of inductors at the input and the output stage. The spiral inductor at the input stage of the LNA is replaced with a suitable active inductor. The gain and Noise Figure of all the three stages depends on the Active inductor. The gain of the Active Inductor (AI) based LNA varies from $25 \mathrm{~dB}$ to $22 \mathrm{~dB}$ throughout the 14 bands of UWB. The noise figure varies from $5 \mathrm{~dB}$ to $6.3 \mathrm{~dB}$. The input impedance lies between $-7 \mathrm{~dB}$ to $-9 \mathrm{~dB}$ and output impedance lies from $-5 \mathrm{~dB}$ to $-14 \mathrm{~dB}$. All simulations are carried out using Advanced Design Software
\end{abstract} (ADS) 2013.

\section{General Terms}

UWB, LNA, Active inductor, OFDM, ADS.

\section{Keywords}

High Quality factor, Noise figure, gain flatness, multiple bands.

\section{INTRODUCTION}

Ultra-wideband is a short range radio technology which is used at a low energy level. It occupies high-bandwidth by using a large portion of the radio spectrum. The maximum output power allowed currently by Federal Communications Commission (FCC) regulations for UWB is $0.0001 \mathrm{~mW} / \mathrm{MHz}$ [1]. Applications of UWB include non-cooperative radar imaging, target sensor data collection, precision locating and tracking applications.

The two different types of UWB technology are: Impulse Radio (IR) UWB technology and Multi Band Orthogonal Frequency Division Multiplexing (MB-OFDM) UWB technology. In impulse radio UWB technology, pulses of very short duration (typically in the order of sub-nanosecond) are transmitted. The spectrum of the bandwidth is several $\mathrm{GHz}$ because of the pulses, which are of very short duration. The IR based UWB technology is a carrier-less transmission. This technology transmits low power since the narrowness of the transmitted pulses has a very fine time resolution. The implementation of this technique is very simple, since no mixer is required. Thus, UWB technology based on IR technology is a low-complexity radio technology as it is carrier-less and needs no mixer. The main disadvantages of the IR-UWB technology are the following: very large bandwidth which results in a bigger challenge in building RF circuits, High-speed Analog to Digital converters and Digital to Analog converters are needed to process the extremely large bandwidth, In multipath environments, multipath energy is not well captured and the sensitivity to group delay variations is large, as the pulse duration is very short

Multi-band Orthogonal Frequency Division Multiplexing (MB-OFDM) is another UWB technology which uses the OFDM method for transfer of information. Multi-Band OFDM combines the OFDM technique with the multi-band approach. The complete UWB spectrum is divided into several sub-bands with a $-10 \mathrm{~dB}$ bandwidth of at least 528 $\mathrm{MHz}$ each. The information is then interleaved across the subbands and then transmitted through multiple carrier (OFDM) technique. The UWB spectrum of 3.1 and $10.6 \mathrm{GHz}$ is divided into 14 sub-bands with minimum of $528 \mathrm{MHz}$ bandwidth that may be added or dropped depending upon the interference from, or to, other systems [2]. The MB-OFDM radio is specifically used in many applications such as WiMax, WPAN for its long range communication [3]. The advantages of the MB-OFDM technique are summarized below: effective capturing of multipath energy with a single RF chain, insensitivity to group delay variations, ability to deal with narrowband interference at receivers and simplified synthesizer architectures relaxing the band switching timing requirements. By all these advantages, MB-OFDM technique proves to be better than the pulse based UWB approach. Therefore, in this paper, an active inductor based LNA is designed for information transfer using MB_OFDM technique. 


\section{LITERATURE SURVEY ON LOW NOISE AMPLIFIER FOR MB-OFDM}

In [5], Zhanfa liu presented a paper on Reconfigurable Multiband LNA in the year 2011. In that paper, he proposed an LNA with voltage gain up to $19 \mathrm{~dB}$ with a Noise figure of 1.9-4.5 dB and with a power consumption less than $30 \mathrm{~mW}$ under $1.8 \mathrm{~V}$ power supply. In this paper, the reconfigurable technique is achieved by means of multi tapped inductor. The operating frequency range varies from $1 \mathrm{GHz}$ to $2.8 \mathrm{GHz}$. The achievement of reconfigurability by making use of multi tapped inductors has also been presented in [6] by Anh Tuan Phan. The other paper on "A 2-11 GHz Reconfigurable multi mode LNA IN $0.13 \mu \mathrm{m}$ CMOS" in 2012 discusses about the multi mode operation of LNA by incorporating a switched multi tap transformer into the input matching network. In this paper, the frequency range is from $4.3 \mathrm{GHz}$ to $10.8 \mathrm{GHz}$. The power dissipation is $6.4 \mathrm{~mW}$ from a $1.2 \mathrm{~V}$ supply, Gain varies from $3 \mathrm{~dB}$ to $15.6 \mathrm{~dB}$; noise figure varies from 4-5.3 dB [7]. But the usage of multi tapped inductors results in inductance leakage and switched inductors occupy more area.

Richard Bierae in 2012 proposed a highly reconfigurable single ended low noise amplifier for Software Defined Radio Application. A variable bias active shunt feedback input matching with switched capacitive load is used to achieve the degree of reconfigurability. This LNA is single ended and operated in the $1.2 \mathrm{~V}$ power supply. The noise figure obtained is in the range of $2.5 \mathrm{~dB}$ and input return loss less than $-10 \mathrm{~dB}$ [8]. In 2013, Ahmed Ragheb presented a paper [9] which includes the design of a reconfigurable LNA for MB-OFDM UWB receivers. In this paper, current reuse architecture has been used in the output stage to achieve reconfigurability.

In the survey on Tunable RF Filters in 2011, they discuss the advantages of the multi-standard wireless systems to the reconfigurable solutions and also discuss the applications towards the 3G multimode devices and their tunable techniques [10]. In [11] and [12] active inductor has been used for the purpose of achieving reconfigurability. Active Inductors has several advantages like high quality factor, less area, tunable inductance value etc. when compared with the previous techniques used in the literature survey.

In this paper, a reconfigurable LNA is achieved with the help of active inductor at the input stage and a spiral inductor at the output stage, making the LNA to operate in fourteen bands of UWB.

\section{PROPOSED LNA WITH ACTIVE INDUCTOR}

The Low Noise Amplifier proposed here is reconfigurable to 14 sub bands of UWB. This reconfigurable LNA is designed for high performance gain and low noise figure. The three stage of LNA comprises of common gate configuration as an input stage, inductive peaking as a core stage and the buffer at the output stage with the unity gain. The proposed LNA is shown in Figure 1

\subsection{Input Stage}

In the input stage (common gate amplifier), the input impedance is $1 / \mathrm{g}_{\mathrm{m}}$. The small signal equivalent of common gate stage is shown in figure 2. The parasitic capacitance $\mathrm{C}_{\mathrm{gs} 1}$ is responsible of poor input matching at higher frequencies. So, the inductor $\mathrm{L}_{1}$ is added to obtain better input matching at the resonant frequency, which is given by equation 2 . The input impedance is given by the equation 1 .

$$
\begin{aligned}
& Z_{\text {in }} \approx \frac{s L_{1}}{1+\left(s C_{g s 1}+g_{m 1}\right) s L_{1}} \\
& \omega_{S}=\frac{1}{\sqrt{C_{g s 1} L_{1}}}
\end{aligned}
$$

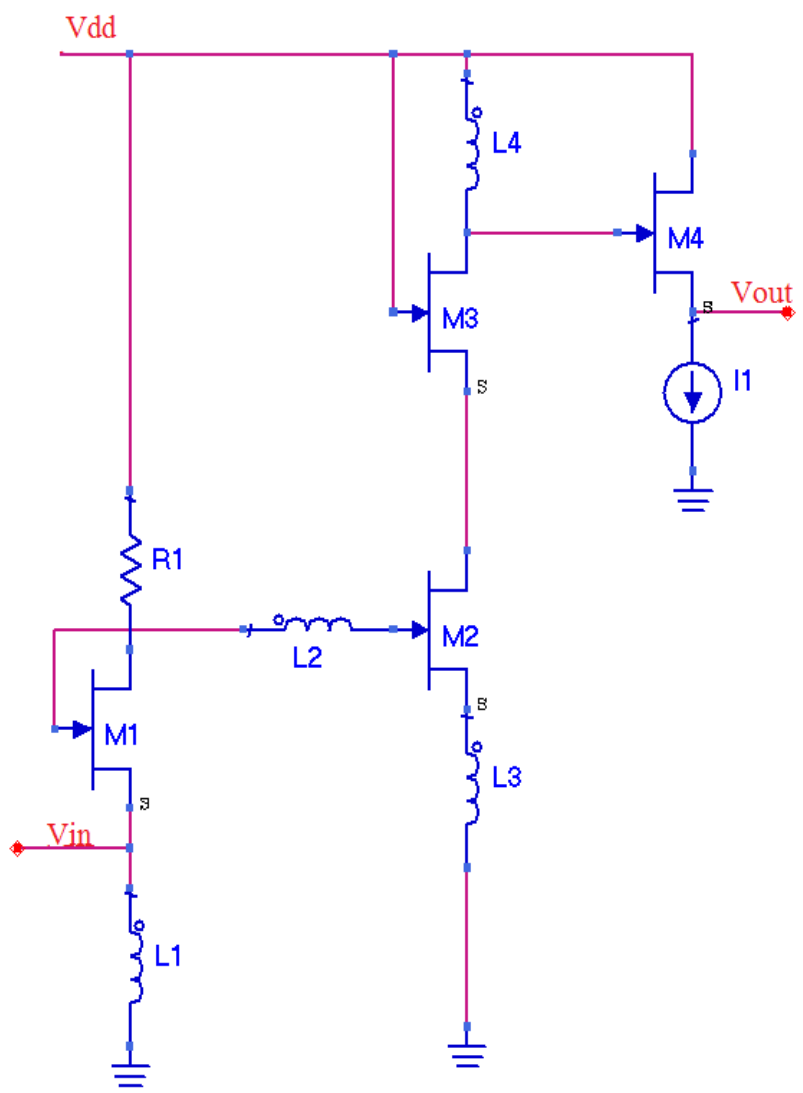

Fig 1: Proposed LNA with Active Inductor at $L_{1}$

The gain achieved by the input stage is derived from the small signal equivalent and it is given in equation 3 .

$$
A_{V 1} \approx g_{m 1} R_{1}
$$

The noise factor is given by the equation 4 . From the derivation, it can be seen that the noise figure is inversely proportional to $\mathrm{g}_{\mathrm{m} 1}$.

$$
N F=1+\frac{\gamma}{g_{m 1} R_{S}}+\frac{R_{S}}{R_{1}}\left(1+\frac{1}{g_{m 1} R_{S}}\right)^{2}
$$

The resonant frequency is fixed at a suitable band of interest by selecting the values of $\mathrm{C}_{\mathrm{gs} 1}$ and $\mathrm{L}_{1}$. This assures good input matching without degrading the value of NF and power gain. By properly selecting the values of $L_{1}$ and the width of $M_{1}$, the input matching of $50 \Omega$ can be easily achieved at the input stage.

\subsection{Core Stage}

The second stage of the LNA is Core stage; it comprises of two transistors $\mathrm{M}_{2}$ and $\mathrm{M}_{3}$. The equivalent circuit of the core stage is given in the Figure 3. This has the effect of providing increased voltage gain of the LNA, provides better output impedance matching and provides shielding as well. The cascode device consists of a transistor $\mathrm{M}_{3}$ which is in 
common-gate configuration. It is normal to set the W/L (channel width versus length) of the Cascode device with the same W/L ratio as the first single-stage of the LNA. In the design of a cascoded LNA, NF and linearity of a LNA are directly affected by the gate width of transistors $\mathrm{M}_{2}$ and $\mathrm{M}_{3}$ and also by the Vgs of the common-source transistor. The noise performance is dominated by the transistor $\mathrm{M}_{2}$. However, linearity performance as well as the improvement of the reverse isolation due to high output impedance are contributed by the transistor $\mathrm{M}_{2}$. The gain of the cascode LNA is given by the equation 5

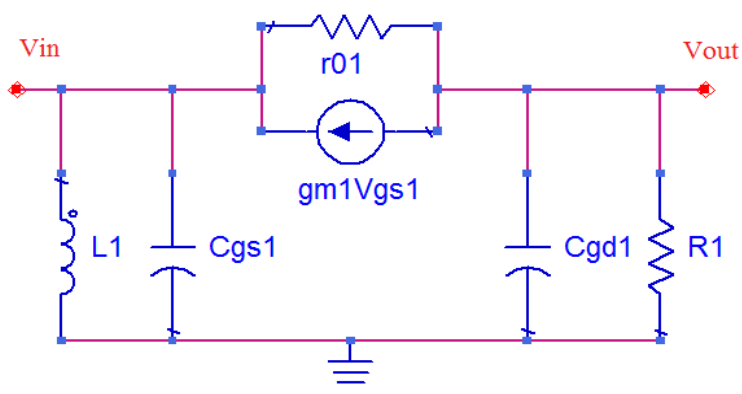

Fig 2: Small Signal Equivalent of CG Stage.

$$
A v_{2}=-g_{m 1} g_{m 2} C_{g s 2} R_{3}
$$

From equation 5 , it can be seen that the gain is high when $\mathrm{g}_{\mathrm{m} 1}=\mathrm{g}_{\mathrm{m} 2}$, that is, the gain will be doubled when compared to common source configuration. Further, the output impedance of cascode stage is high resulting in increasing gain and hence cascode configuration is widely used as a core stage when compared to CS configuration. The noise in cascode stage is dominated by the first stage and hence NF is given by the equation 6 ,

$$
N F=1+\frac{2}{3} \frac{1}{g_{m 1} g_{m 2} R_{S}^{2}}+\left(f / f_{t}\right)^{2} \frac{2}{3} \frac{1}{g_{m 1} g_{m 2} R_{S}}
$$

The NF equation shows that if the transconductance $g_{\mathrm{m} 1}$ and $\mathrm{g}_{\mathrm{m} 2}$ increases, then NF decreases. A good matching is achieved by the network, formed by the resistor $\mathrm{R}_{1}$ and the inductor $\mathrm{L}_{1}$.

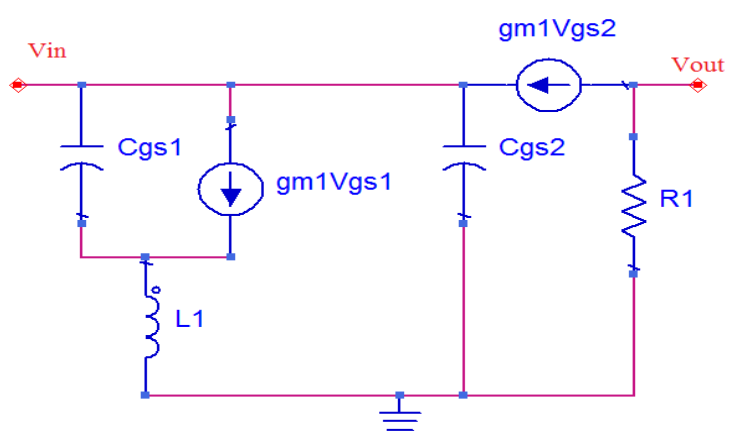

Fig 3: Small Signal Equivalent of Core Stage.

\subsection{Output Stage}

The output matching networks are used to match the output of the LNA to the next stage in the receiver. A good output matching network should provide low noise to the output, good output matching with less reflection, good gain and bandwidth. A Common drain (CD) configuration suits this purpose. The $\mathrm{CD}$ configuration small signal equivalent is shown in figure 4 . The $\mathrm{CD}$ configuration is not used a core stage as its gain is always less than unity, which is shown by the equation 7 ,

$$
A_{V 3}=\frac{g_{m 4} r_{04}}{1+g_{m 4} r_{04}}
$$

Where $r_{04}$ is the internal resistance and $g_{m 4}$ is the transconductance of $\mathrm{M}_{4}$. The output impedance is given by equation 8 , and hence it is normally used for output matching. The NF is given below, by equation 9 .

$$
\begin{aligned}
& Z_{\text {out }}=R_{\text {O4 }} \\
& N F=1+\frac{\gamma\left(1 / g m_{4}\right)}{R_{S}\left(g_{m 4} r 04 /\left(1+g_{m 4} r 04\right)\right.}{ }^{2}
\end{aligned}
$$

Thus, the three stages of the proposed LNA and its design equations are discussed. The overall gain of the LNA is given by multiplication of the gains mentioned in equations 3,5 and 7.

Here, the spiral inductor $\mathrm{L}_{1}$, which is responsible for input matching, is replaced by the active inductor, thereby achieving multiple bands in the UWB band.

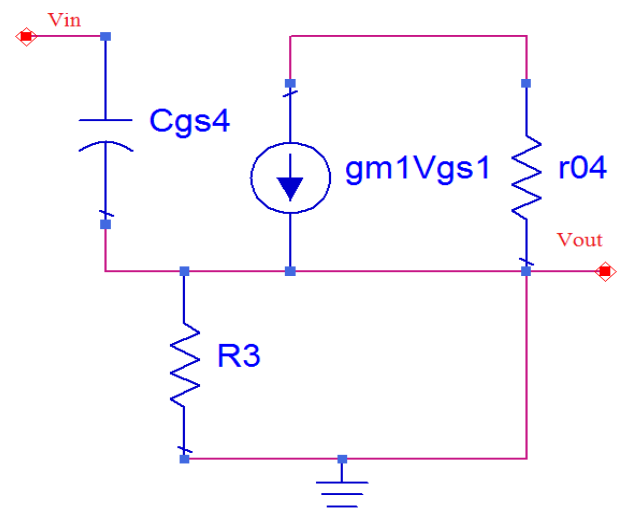

Fig 4: Small Signal Equivalent of output Stage.

\subsection{Component Values}

The following table lists the component values of the inductors used to achieve multiband. Inductors $\mathrm{L}_{2}$ and $\mathrm{L}_{3}$ are fixed with a value of $1 \mathrm{nH}$ and $0.5 \mathrm{nH}$ respectively. The inductance $\mathrm{L}_{1}$ is replaced by an active inductor which is tunable for fourteen bands and $\mathrm{L}_{4}$ is a spiral inductor. The value of $\mathrm{L}_{4}$ is also varied throughout the UWB band and the component values of the inductors are listed in table below.

\section{PROPOSED ACTIVE INDUCTOR}

In this paper, an AI has been proposed, in order to provide a high Quality factor and low noise figure. Thermal noise cancellation has been done in the proposed $\mathrm{AI}$, which is explained in figure 5. Here the NMOS transistors $M_{1}$ and $M_{2}$ form the positive transconductance and the PMOS transistors $\mathrm{M}_{3}$ and $\mathrm{M}_{4}$ forms the negative transconductance of the Active Inductor. The noise current of $\mathrm{M}_{2}$ flows into the node 2 but out of node 3 . Thus two fully correlated noise voltages with opposite phases are produced at nodes 2 and 3 as shown in figure 5 . These two voltages are converted into current by the $\mathrm{M}_{3}$ and $\mathrm{M}_{4}$ transistors respectively. At resonant frequency, by properly matching the AC currents of $\mathrm{M}_{3}$ and $\mathrm{M}_{4}$, the noise contributed by the transistor $\mathrm{M}_{2}$ can be cancelled at the input. Conversely, the signal voltages at nodes 2 and 3 are in phase, resulting in constructive addition at node 1 , as shown in figure 5. This structure results in a very low NF [13]. In addition to 
this less NF, the $\mathrm{Q}$ factor of the AI can be improved by adding the RC structure as feedback [14]. The current source $\left(\mathrm{I}_{1}\right)$ and sink $\left(\mathrm{I}_{2}\right.$ and $\left.\mathrm{I}_{3}\right)$ used in figure 5 are implemented using the regulated cascode structure. We go for the regulated cascode structure, because it provides stable current over a wide range of temperature. In [15], Zhangming Zhu et al proposed a low voltage CMOS cascade current mirror based on bulk driven PMOS transistor (BDCCM). This structure has been replaced in place of ideal current source in figure 5 and the ideal current sink has been replaced by regulated cascade current sink.

Table 1. Component Values

\begin{tabular}{|c|c|c|}
\hline Bands & $\mathrm{L}_{1}(\mathrm{nH})$ & $\mathrm{L}_{4}(\mathrm{nH})$ \\
\hline Band 1 & 8 & 12.2 \\
\hline Band 2 & 7.8 & 10.5 \\
\hline Band 3 & 7.5 & 8.5 \\
\hline Band 4 & 7 & 7.3 \\
\hline Band 5 & 6.8 & 4.9 \\
\hline Band 6 & 6.2 & 4.5 \\
\hline Band 7 & 5.7 & 3.8 \\
\hline Band 8 & 5.5 & 3.3 \\
\hline Band 9 & 5.2 & 2.8 \\
\hline Band 10 & 5 & 2.4 \\
\hline Band 11 & 4.5 & 2.2 \\
\hline Band 12 & 4 & 2.2 \\
\hline Band 13 & 3.5 & 2.06 \\
\hline Band 14 & 3 & 1.65 \\
\hline
\end{tabular}

The proposed AI is always lossy. This lossy inductor can be modeled with the help of parasitic resistances and capacitances [16]. These resistances and capacitances are either modeled as a series RLC or parallel RLC circuit. Here, we stick on to parallel RLC model. The gyrator-C realization of the $\mathrm{AI}$ and its equivalent circuit model is shown in figure 6 . Here the transistors $\mathrm{M}_{1}$ and $\mathrm{M}_{2}$ form the positive transconductance $\mathrm{Gm}_{1}$ and the transistors $\mathrm{M}_{3}$ and $\mathrm{M}_{4}$ forms the negative transconductance $\mathrm{Gm}_{2}$.

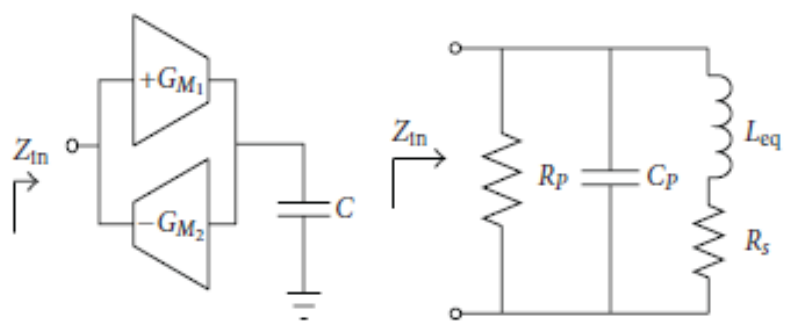

Fig 6: Gyrator $\mathrm{C}$ realization and its equivalent circuit model [16].

The above figure shows the gyrator realization of active inductor, which is formed by connecting two transconductance back to back. The values of $\mathrm{R}, \mathrm{L}$ and $\mathrm{C}$ in the equivalent circuit are derived by finding the input impedance of the AI shown in figure 5. Their values are given by equations 10 to 13 . The resonant frequency of the $\mathrm{AI}$ is defined by equations 14 and 15 and the Quality factor of a parallel RLC circuit is given by equation 16 .

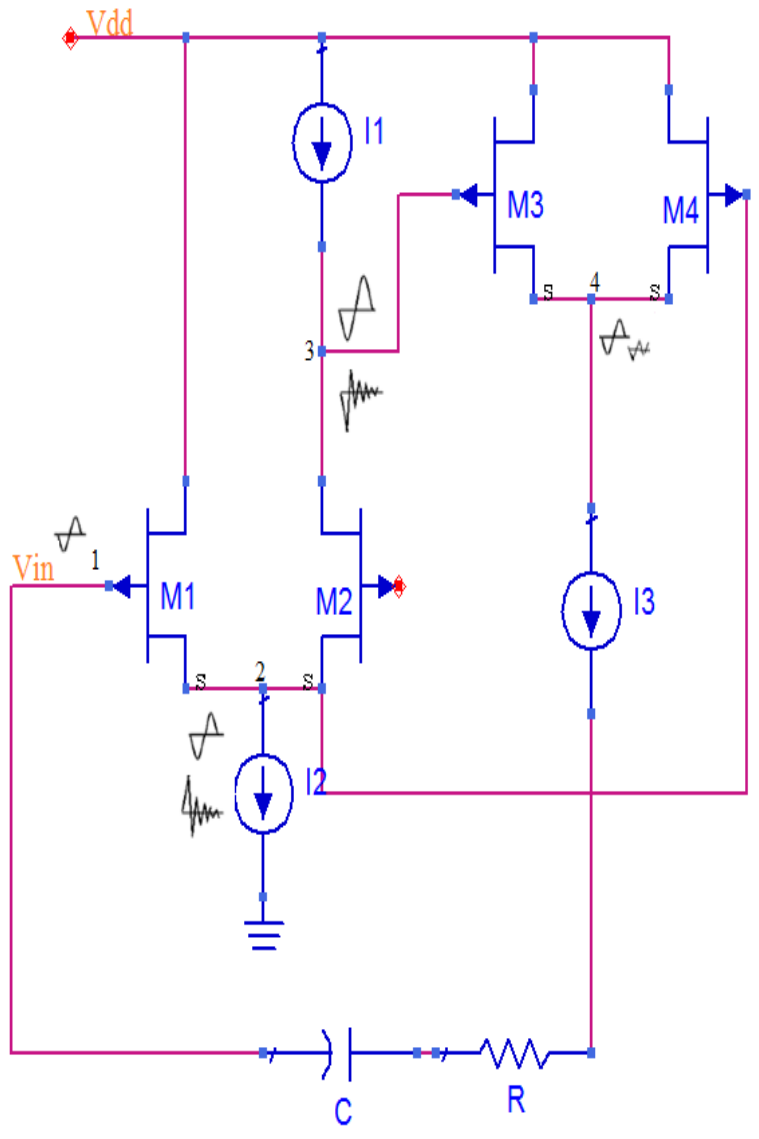

Fig 5: Proposed AI

$$
\begin{aligned}
& L s \approx \frac{\left(G m C_{3}\left(g_{f}+\frac{1}{g_{c}}+g_{4}\right) /\left(g_{f}+\frac{1}{g_{c}}\right)\right.}{\left(g m_{1} g m_{2} g m_{3}+g m_{1} g m_{4}+g_{3}\right)} \\
& R s \approx \frac{G m g_{3} /\left(\left(g m_{1} g m_{2} g m_{3}+g m_{1} g m_{4}+g_{3}\right)\right.}{\left(g_{f}+\frac{1}{g_{c}}+g_{4}\right)} \\
& C p \approx C g s_{1} \\
& R s \approx 0 \\
& f_{0}=\frac{1}{2 \pi \sqrt{L_{s} C_{p}}} \\
& \omega_{0} \approx \sqrt{\frac{g m_{1} g m_{2} g m_{3}+g m_{1} g m_{4}+g_{3}}{\left.G m C_{1} C_{3}\left(g_{f}+\frac{1}{g_{c}}\right) /\left(g_{f}+\frac{1}{g_{c}}+g_{4}\right)\right)}} \\
& Q=R p / \omega L s
\end{aligned}
$$

Where $\mathrm{g}_{\mathrm{m}}=\mathrm{g}_{\mathrm{m} 1}+\mathrm{g}_{\mathrm{m} 2}+\mathrm{g}_{\mathrm{m} 3}$. 


\section{SIMULATION RESULTS AND DISCUSSION}

The simulation results of AI and LNA are discussed in this section. For Active Inductor, parameters like Inductance, Quality factor and noise figure has been simulated. The parameters of LNA like power gain, NF, input matching and output matching are plotted for fourteen bands and the results are discussed below

\subsection{Simulation Results of AI}

Q-factor defines the maximum amount of energy stored in the inductor. For a good inductor, Q-factor should be very high. For the proposed AI we get a maximum quality factor of 5500 at $8.9 \mathrm{GHz}$ resonant frequency, which is shown in figure 7 .

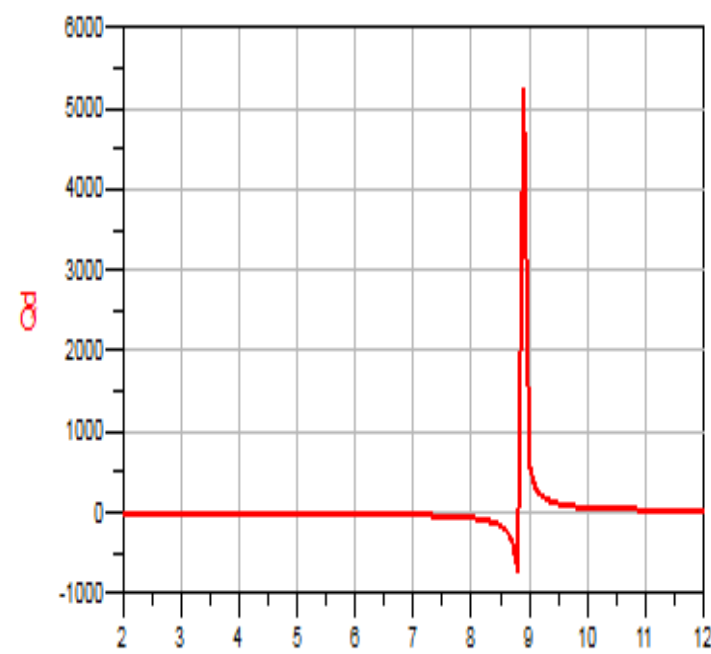

Fig 7: Q-factor of AI

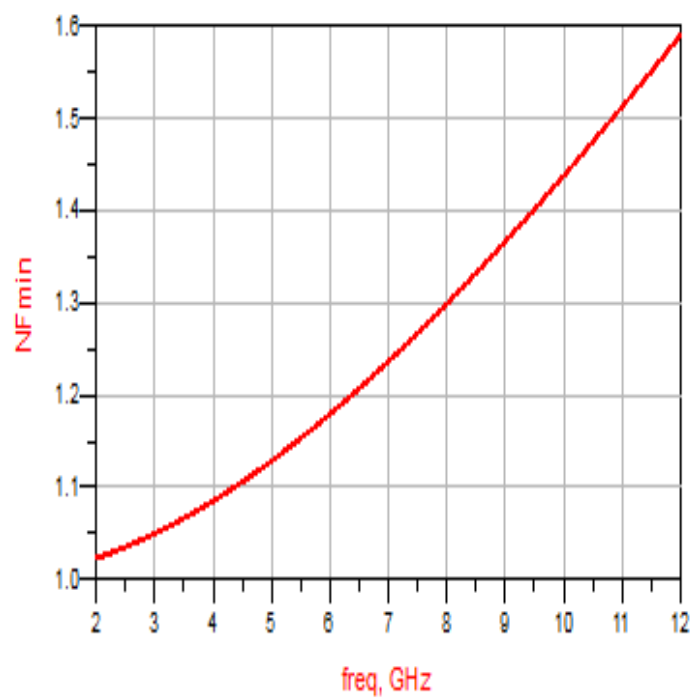

Fig 8: NF of AI

The noise figure of the active inductor is shown in figure 8 . The noise figure varies from $1 \mathrm{~dB}$ to $1.6 \mathrm{~dB}$ in the UWB band, thereby reducing the noise figure of the LNA also, when introduced at its input stage. The inductance value of the active inductor varies from $9 \mathrm{nH}-3 \mathrm{nH}$ in the entire UWB band. This active inductor is responsible for achieving multiband in the entire UWB frequency band. The plot of inductance value is shown in figure 9 .

\subsection{Simulation Results of LNA}

The power gain of LNA is given by the parameter S21. For the LNA to have better performance, power gain should be high. The gain for fourteen bands varies from 24 to $22 \mathrm{~dB}$ and is shown in figure 13. It can be seen from the results that the introduction of $\mathrm{AI}$ also results in gain flatness.

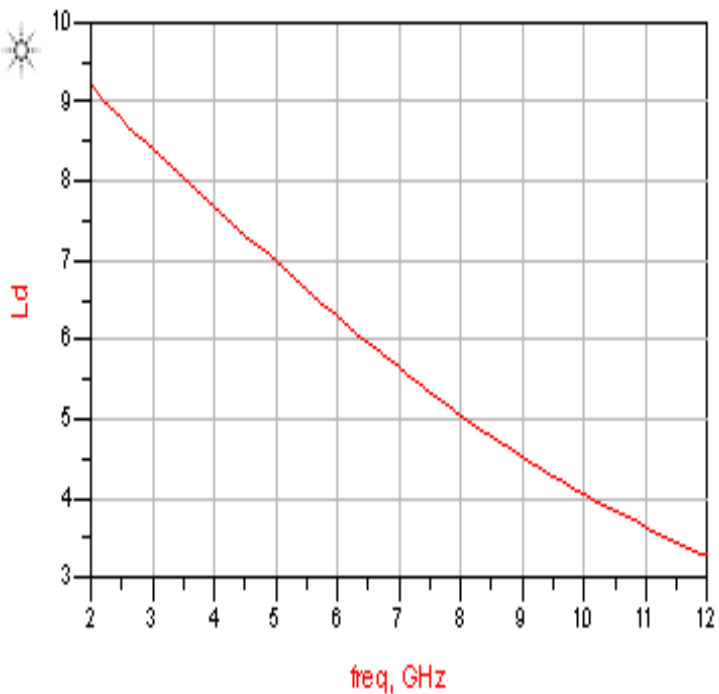

Fig 9: Inductance value of AI
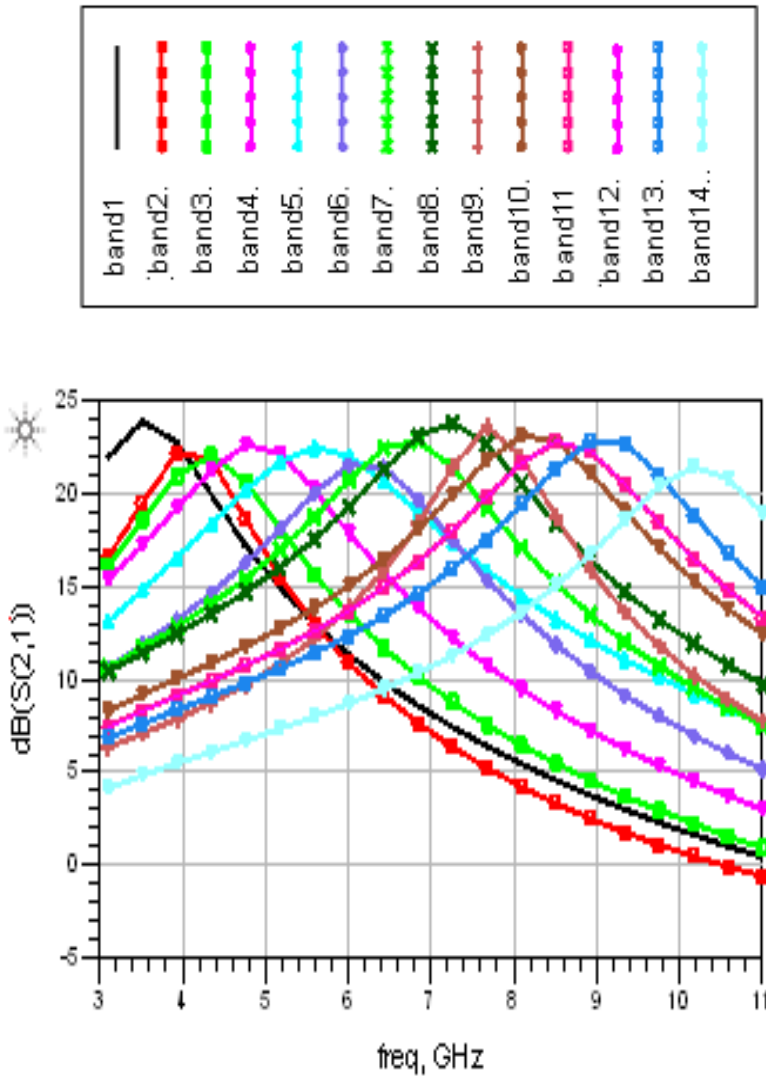

Fig 9: Power gain of LNA

The noise figure of the LNA varies from $5 \mathrm{~dB}$ to $6.5 \mathrm{~dB}$ in the fourteen bands, as shown in figure 14. The noise figure of the LNA is mainly contributed by the active inductor at the input stage. Since the noise figure of the Active Inductor is only around $1.5 \mathrm{~dB}$, the noise figure of the LNA is quiet lesser throughout the fourteen bands. The input impedance reflection 
coefficient S11 and the output impedance reflection coefficient S22 for the fourteen bands are as shown in figure 11 and figure 12 respectively. S11 is less than $-5 \mathrm{~dB}$ and $\mathrm{S} 22$ is also less than $-5 \mathrm{~dB}$.

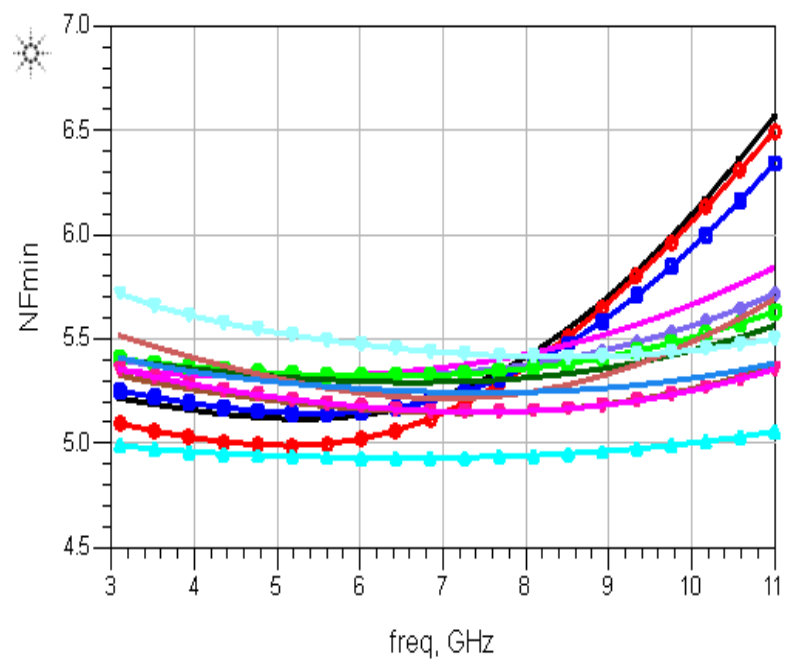

Fig 10: NF of LNA

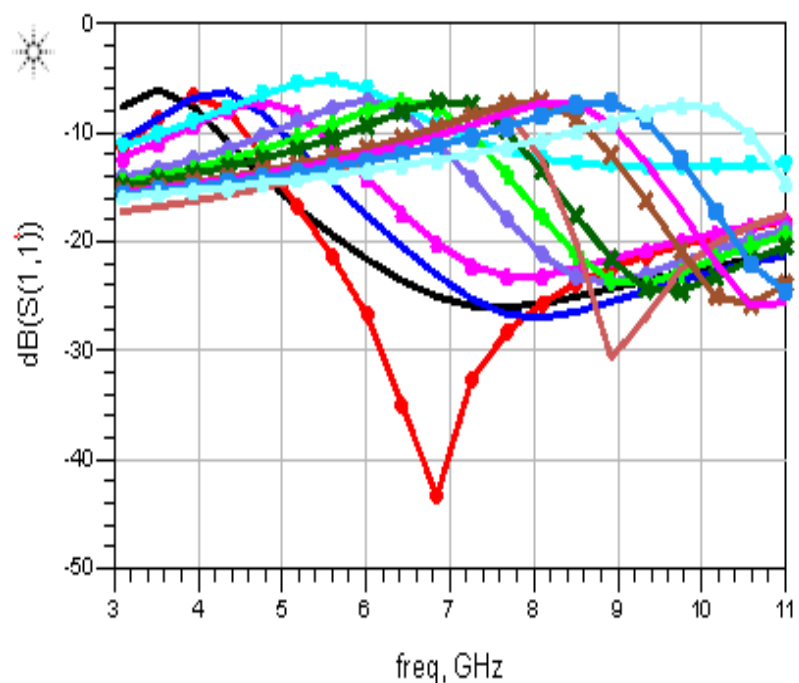

Fig 11: Input reflection coefficient of LNA

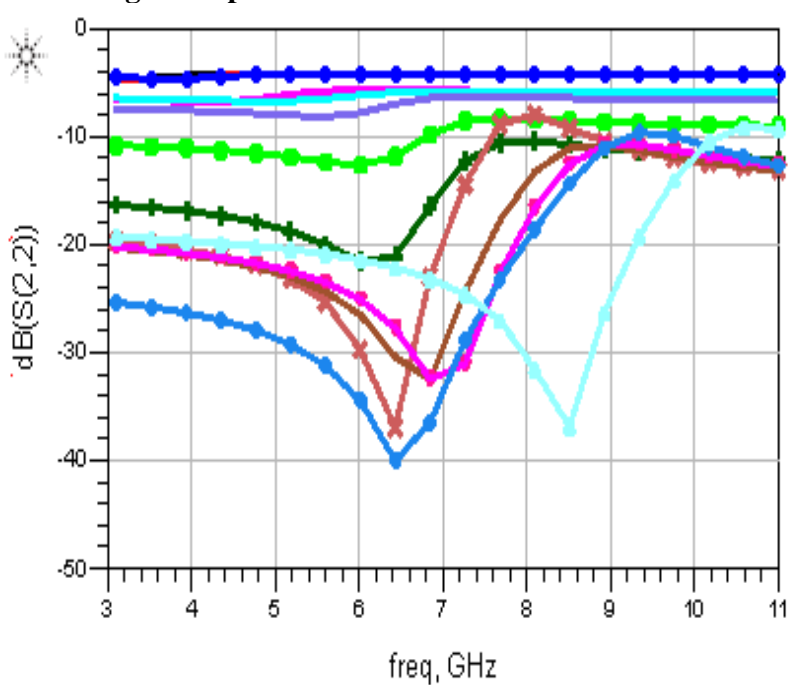

Fig 12: Output reflection coefficient of LNA
The summary of this simulation results are shown in table 2 .

Table 2. Simulation results

\begin{tabular}{|c|c|c|c|c|c|}
\hline $\begin{array}{c}\text { Band } \\
\text { No }\end{array}$ & $\begin{array}{c}\text { Bandwidth } \\
\text { (MHz) }\end{array}$ & $\begin{array}{l}\text { S21 } \\
(\mathrm{dB})\end{array}$ & NFmin & $\begin{array}{l}\text { S11 } \\
\text { (dB) }\end{array}$ & $\begin{array}{l}\mathrm{S} 22 \\
(\mathrm{~dB})\end{array}$ \\
\hline 1 & $3168-3696$ & 24 & 5.3 & -5 & -5 \\
\hline 2 & $3696-4224$ & 22 & 5.4 & -7 & -5 \\
\hline 3 & $4224-4752$ & 22 & 5.4 & -5 & -5 \\
\hline 4 & $4752-5280$ & 23 & 5.5 & -5 & -8 \\
\hline 5 & $5280-5808$ & 23 & 5.4 & -5 & -9 \\
\hline 6 & $5808-6336$ & 22 & 5.5 & -8 & -9 \\
\hline 7 & $6336-6864$ & 23 & 5.6 & -9 & -12 \\
\hline 8 & 6864-7392 & 24 & 5.7 & -6 & -14 \\
\hline 9 & $7392-7920$ & 22 & 5.7 & -6 & -10 \\
\hline 10 & $7920-8448$ & 23 & 5.8 & -7 & -12 \\
\hline 11 & $8448-8976$ & 23 & 5.9 & -6 & -13 \\
\hline 12 & $8976-9504$ & 23 & 6 & -6 & -12 \\
\hline 13 & $\begin{array}{l}9504- \\
10032\end{array}$ & 23 & 6.2 & -9 & -12 \\
\hline 14 & $\begin{array}{c}10032- \\
10560\end{array}$ & 22 & 5.9 & -12 & -12 \\
\hline
\end{tabular}

\section{CONCLUSION}

This project mainly discusses about the gain improvement, bandwidth extension and the area compaction of the Low Noise amplifier for MB-OFDM applications. This paper majorly deals with the single LNA circuit for the entire 14 bands of MB-OFDM. The AI achieves a very high Quality factor of about 5500, vary low noise figure of around $1.5 \mathrm{~dB}$ in the entire UWB band. The substitution of this active inductor at the input stage of the proposed LNA results in a sufficiently high gain, which lies between $24 \mathrm{~dB}$ to $22 \mathrm{~dB}$. The noise figure varies from $5 \mathrm{~dB}$ to $6.5 \mathrm{~dB}$. The input return loss and the output return loss are less than $-5 \mathrm{~dB}$. All these Simulations were carried out using the software Advanced design system version 2013. This paper also prescribes the importance of active inductor for the LNA, which mainly results in the reduced area and bandwidth extension in the LNA.

\section{REFERENCES}

[1] Roberto Aiello.2006 Ultra Wide Band technologies and applications, Elsevier Publications, ISBN 13: 978-07506-7893-3. pp 2- 10.

[2] Homayoun nikookar. 2009 Introduction to Ultra Wide Band for wireless communications, Springer publications, ISBN: 978-1-4020-6632-0. pp 1- 25. 
[3] Moshe ran and Yossef ben ezra. 2009 Green femtocell based on UWB technologies, www.intechopen.com

[4] Yu-na Su and Geng Li . 2012 Design of a Low Noise Amplifier of RF Communication Receiver for Mine, IEEE symposium on Electrical and Electronics engineering,

[5] Zhanfa liu and Jing wang. 2011 A $0.18 \mu \mathrm{m}$ CMOS Reconfigurable Multiband multi gain Low Noise Amplifier, IEEE proceedings in RF systems.

[6] Anh Tuan Phan and Ronan Farrell. 2010 "Reconfigurable Multiband Multimode LNA for LTE/GSM, WiMAX, and IEEE 802.11.a/b/g/n", IEEE.

[7] Xiaohua Yu and Nathan M. Neihart. 2012 A 2-11 GHz Reconfigurable multi mode LNA IN $0.13 \mu \mathrm{m}$ CMOS, IEEE symposium on Radio frequency Integrated circuits.

[8] Richard Beare, Calvin Plett and John Rogers. 2012. Highly Reconfigurable single ended low noise amplifier for Software Defined Radio application, IEEE conference on Microsystems.

[9] Ahmed Ragheb. 2013 A Novel Reconfigurable MB-OFDM UWB LNA Using Programmable Current Reuse, International Journal of Microwave Science and Technology 02/2013.

[10] Abubakar Sadiq Hussaini, Raed Abd-Alhameed and Jonathan Rodriguez. 2012 "Tunable RF
Filters:- Survey and beyond", IEEE journal of RF filter

[11] V. Vaithianathan, J.Raja and R.Srinivasan. 2012 A $1.7-2.5 \mathrm{GHz}$ Active Inductor based Low Power Low Noise Amplifier for Multi Standard Applications, International Journal of Computer Applications, Volume-39, and No.5.

[12] J. Manjula and S. Malarvizhi. 2012 Low Power Low Noise Tunable Active Inductor for Narrow Band LNA Design, International Journal of Computer Applications, Volume-47, No.17.

[13] Santosh Vema Krishnamurthy, Kamal El-Sankary, and Ezz El-Masry. 2010. Noise-Cancelling CMOS Active Inductor and Its Application in RF BandPass Filter Design, International Journal of Microwave Science and Technology Volume, Article ID 980957.

[14] Rafei. 2012 Cancellation of Series Loss Resistance in Ultra Wideband Inductor Using RC Feedback, Iranian Journal, Vol. 8, No. 2

[15] Zhangming Zhu , Jianbin MO and Yintang Yang. 2008 A Low Voltage Bulk-driving PMOS Cascode Current Mirror, IEEE.

[16] Fei Yuan. 2008 CMOS Active Inductors and Transformers, ISBN 978-0-387-76477-1, Springer publications. 